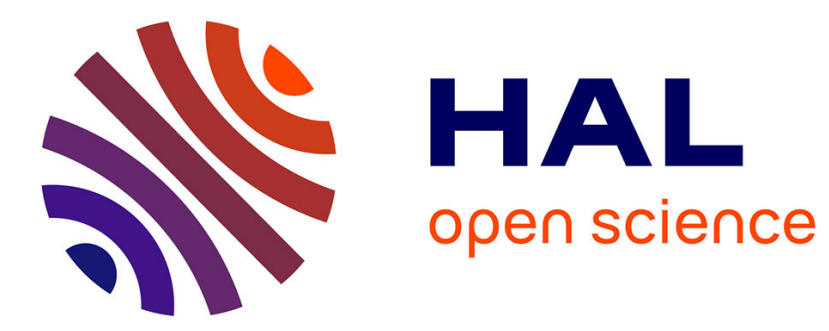

\title{
Failure in shear bands for granular materials: thermo-hydro-chemomechanical effects
}

Emmanuil Veveakis, Ioannis Stefanou, Jean Sulem

\section{To cite this version:}

Emmanuil Veveakis, Ioannis Stefanou, Jean Sulem. Failure in shear bands for granular materials: thermo-hydro-chemomechanical effects. Géotechnique Letters, 2013, 3 (2), pp.31-36. 10.1680/geolett.12.00063 . hal-00862508

\section{HAL Id: hal-00862508 \\ https://hal.science/hal-00862508}

Submitted on 16 Sep 2013

HAL is a multi-disciplinary open access archive for the deposit and dissemination of scientific research documents, whether they are published or not. The documents may come from teaching and research institutions in France or abroad, or from public or private research centers.
L'archive ouverte pluridisciplinaire HAL, est destinée au dépôt et à la diffusion de documents scientifiques de niveau recherche, publiés ou non, émanant des établissements d'enseignement et de recherche français ou étrangers, des laboratoires publics ou privés. 


\title{
Failure in shear bands for granular materials: thermo-hydro-chemo- mechanical effects
}

\author{
M. VEVEAKIS*, I. STEFANOU $\dagger$ and J. SULEM $\dagger$
}

\begin{abstract}
The failure of geomaterials in localised shear bands is one of the most common features in geomechanics. Early studies have provided the necessary criteria for the conditions of localisation, inclination angle with respect to the loading axes and thickness of the shear bands, when loaded at room temperature. This work extends these criteria for the problem of simple shear of a chemically active granular cohesionless material at higher temperatures, where thermal or chemical pressurisation may set in and influence the response of the material. It is deduced that failure occurs at higher positive values of the hardening modulus as temperature increases, that the shear band thickness also depends on the chemical reaction characteristics and that micro-inertia due to grain translations and rotations introduce the necessary rate dependency to regularise the system.
\end{abstract}

KEYWORDS: chemical properties; plasticity; temperature effects

ICE Publishing: all rights reserved

\section{INTRODUCTION}

In classical continuum mechanics, where the continuum is allowed only for translational degrees of freedom (displacements), one may be able to predict the onset of a shear band (Rudnicki \& Rice, 1975). However, it can be shown that the instability tends to localise in a strip of zero thickness. This drawback of the classical theories is attributed to the fact that they do not contain material parameters with dimensions of length that can scale the shear band thickness (internal length).

To compensate for this inability, Hill (1962) and Mandel (1966) in their early approaches artificially imposed a failure structure with width $D$, thus suggesting that the solutions obtained from classical continua correspond to the limit $D \rightarrow 0$ of a corresponding higher grade extension of it (Vardoulakis \& Sulem, 1995). Following this conclusion, as well as experimental observation of the influence of the effect of particle irregularities and rotations inside the shear bands, Muhlhaus \& Vardoulakis (1987) resorted to concepts from Cosserat continuum mechanics to naturally determine $D$. Thus, by allowing both particle displacements and particle rotations, they enriched the classical continuum with additional kinematic and static fields, and calculated that $D \sim 16 d_{50}$ for sands.

In this work, the concept of the Cosserat continuum is extended by using it in the problem of simple shear of a saturated, chemically active, granular cohesionless material. The effect of temperature on both the conditions of failure and shear band thickness is studied.

\section{PROBLEM FORMULATION}

Consider a layer of saturated granular material of thickness $D$ that is sheared in plane strain (Fig. 1) in such a way that

Manuscript received 17 December 2012; first decision 7 February 2013; accepted 9 April 2013.

Published online at www.geotechniqueletters.com on 24 May 2013.

${ }^{*}$ CSIRO Earth Science and Resource Engineering, Kensington, WA, Australia

†UR Navier-CERMES, Ecole des Ponts ParisTech, Université Paris-Est, Marne-la-Vallée, France there is no extensional strain in the $x_{1}$-direction and displacements $u_{1}$ and $u_{2}$ of material points vary only with $x_{2}$ (and time $t$ ). The additional rotational degree of freedom of the considered two-dimensional (2D) Cosserat continuum is $\omega_{\mathrm{c}}$.

\section{Constitutive equations of elasto-plasticity}

The incremental constitutive equations for the considered 2D Cosserat continuum are derived from Muhlhaus \& Vardoulakis (1987). They are briefly recalled in the following.

In a $2 \mathrm{D}$ Cosserat continuum, each material point has two translational $\left(v_{1}, v_{2}\right)$ and one rotational degree of freedom $\dot{\omega}_{\mathrm{c}}$. The rate of the non-symmetric deformation tensor is given as

$$
\begin{aligned}
& \dot{\epsilon}_{11}=\frac{\partial v_{1}}{\partial x_{1}} \\
& \dot{\epsilon}_{12}=\frac{\partial v_{1}}{\partial x_{2}}+\dot{\omega}_{\mathrm{c}} \\
& \dot{\epsilon}_{21}=\frac{\partial v_{2}}{\partial x_{1}}-\dot{\omega}_{\mathrm{c}} \\
& \dot{\epsilon}_{22}=\frac{\partial v_{2}}{\partial x_{2}}
\end{aligned}
$$

and the two components of the curvature of the deformation rate (gradient of the Cosserat rotation rates) are

$$
\begin{aligned}
& \dot{\kappa}_{1}=\frac{\partial \dot{\omega}_{\mathrm{c}}}{\partial x_{1}} \\
& \dot{\kappa}_{2}=\frac{\partial \dot{\omega}_{\mathrm{c}}}{\partial x_{2}}
\end{aligned}
$$

Moreover, we decompose the stresses and strain rates into spherical and deviatoric parts

$$
\sigma_{i j}=s_{i j}+\sigma_{k k} \delta_{i j} / 2
$$

and

$$
\dot{\epsilon}_{i j}=\dot{e}_{i j}+\dot{\epsilon}_{k k} \delta_{i j} / 2
$$




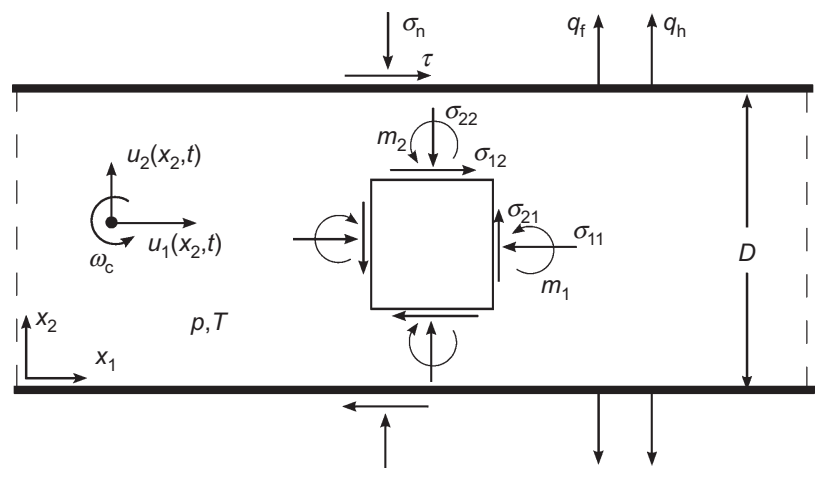

Fig. 1. Problem formulation. Cosserat stresses, couple stresses and degrees of freedom

where $\delta_{i j}$ is the Kronecker delta. The following generalised stress and strain invariants are used

$$
\begin{aligned}
& \sigma=\sigma_{i i} / 2 \\
& \tau=\left(h_{1} s_{i j} s_{i j}+h_{2} s_{i j} s_{j i}+h_{3} m_{k} m_{k} / R\right)^{1 / 2} \\
& \dot{\epsilon}^{\mathrm{p}}=\dot{\epsilon}_{k k}^{\mathrm{p}} \\
& \dot{\gamma}^{p}=\left(g_{1} \dot{e}_{i j}^{\mathrm{p}} \dot{e}_{i j}^{\mathrm{p}}+g_{2} \dot{e}_{i j}^{\mathrm{p}} \dot{e}_{j i}^{\mathrm{p}}+R^{2} g_{3} \dot{\kappa}_{k} \dot{\kappa}_{k}\right)^{1 / 2}
\end{aligned}
$$

where $h_{i}=[3 / 4,-1 / 4,1]$ and $g_{i}=[3 / 2,1 / 2,1]$ for the so-called static Cosserat model and $R$ is the internal length (Vardoulakis \& Sulem, 1995), here set equal to the average grain radius. Besides the four components of the nonsymmetric stress-tensor $\sigma_{i j}$, there are two couple stresses $m_{k}$ $(k=1,2)$, shown in Fig. 1 .

As in classical small-strain plasticity theory, the deformation rates $\dot{\epsilon}_{i j}, \dot{\kappa}_{k}$ are decomposed into elastic $\dot{\epsilon}_{i j}^{\mathrm{e}}, \dot{\kappa}_{k}^{\mathrm{e}}$ and plastic parts $\dot{\epsilon}_{i j}^{\mathrm{p}}, \dot{\kappa}_{k}^{\mathrm{p}}$

$$
\begin{aligned}
& \dot{\epsilon}_{i j}=\dot{\epsilon}_{i j}^{\mathrm{e}}+\dot{\epsilon}_{i j}^{\mathrm{p}} \\
& \dot{\kappa}_{k}=\dot{\kappa}_{k}^{\mathrm{e}}+\dot{\kappa}_{k}^{\mathrm{p}}
\end{aligned}
$$

Following Sulem et al. (2011), we assume a Terzaghi decomposition of the stress tensor in effective stress $\sigma_{i j}^{\prime}$ and pore pressure $p\left(\sigma_{i j}=\sigma_{i j}^{\prime}-p \delta_{i j}\right)$ and assume a Coulomb yield stress and plastic potential for the effective stresses

$$
\begin{aligned}
& F=\tau+\mu(\sigma+p) \\
& Q=\tau+\beta(\sigma+p)
\end{aligned}
$$

where the mobilised friction coefficient $\mu$ and the dilatancy coefficient $\beta$ are functions only of the accumulated plastic strain $\gamma^{\mathrm{p}}$. Under these considerations, the rate thermoporo-elasto-plastic relationships are expressed as

$$
\begin{aligned}
& \dot{\gamma}=\frac{\dot{\tau}}{G}+\dot{\gamma}^{\mathrm{p}} \\
& \dot{\epsilon}=\frac{1}{K}(\dot{\sigma}+\dot{p})+\alpha_{\mathrm{s}} \dot{T}+\dot{\epsilon}^{\mathrm{p}} \\
& \dot{\kappa}_{k}=\frac{\dot{m}_{k}}{G R^{2}}
\end{aligned}
$$

where $G$ and $K$ are the elastic shear and bulk modulus, respectively, $\alpha_{\mathrm{s}}$ is the thermal dilation coefficient of the solid skeleton and $T$ is the temperature. The rate of plastic deformation is written as

$$
\dot{\gamma}^{\mathrm{p}}=\frac{1}{H}[\dot{\tau}+\mu(\dot{\sigma}+\dot{p})], \dot{\epsilon}^{\mathrm{p}}=\beta \dot{\gamma}^{\mathrm{p}}
$$

where $H=H\left(\gamma^{\mathrm{p}}\right)=h(\sigma+p)\left(\right.$ with $\left.h=\mathrm{d} \mu / \mathrm{d} \gamma^{\mathrm{p}}\right)$ is the plastic hardening modulus, which is related to the tangent modulus $H_{\tan }$ of the $\tau$ versus $\gamma$ curve through the relationship

$$
H_{\tan }=\frac{H}{1+H / G}
$$

\section{Governing equations}

For the considered 2D Cosserat continuum and the configuration of Fig. 1, the balance of linear and angular momentum provides

$$
\begin{aligned}
& \frac{\partial \sigma_{12}}{\partial x_{2}}=\rho \frac{\partial v_{1}}{\partial t} \\
& \frac{\partial \sigma_{22}}{\partial x_{2}}=\rho \frac{\partial v_{2}}{\partial t} \\
& \frac{\partial m}{\partial x_{2}}+\sigma_{21}-\sigma_{12}=\rho \frac{R^{2}}{2} \frac{\partial \dot{\omega}_{\mathrm{c}}}{\partial t}
\end{aligned}
$$

where $m=m_{2}$ and $m_{1}=0$. The stresses applied at the boundary are a shear stress $\tau$ and a normal stress $\sigma_{\mathrm{n}}$ in the $x_{2}$-direction. It is assumed that no couple stress is imposed at the boundary. Prior to localisation, the state of stress and strain is uniform and, considering the couple-free boundary condition, the couple stress is identically zero in the sheared layer. Therefore, the rock behaves as a classical continuum before localisation.

Let us impose the presence of a first-order decomposition reaction that decomposes the solid skeleton and produces excess pore fluid in a process called chemical pressurisation. As an example, consider calcite decomposition, $\mathrm{CaCO}_{3} \rightarrow \mathrm{CaO}+\mathrm{CO}_{2}$, producing supercritical fluid $\mathrm{CO}_{2}$. The same framework, however, could be generalised for any other decomposition reaction imposing chemical pressurisation. Assuming that the calcite decomposition takes place with a rate $r$, given by

$$
r \approx \frac{\rho_{\mathrm{s}}}{M_{\mathrm{CaCO}_{3}}} A_{0} \mathrm{e}^{-T_{\mathrm{c}} / T}
$$

where $T_{\mathrm{c}}=E / R_{\mathrm{a}}$ is the activation temperature of the reaction, $E$ is the activation energy, $R_{\mathrm{a}}$ is the universal gas constant, $M_{\mathrm{CaCO}_{3}}$ is the molar mass of calcite and $A_{0}$ is the pre-exponential factor. Under these considerations and assuming that the grains are plastically incompressible, the mass balance equation provides (Sulem et al., 2011; Veveakis et al., 2012)

$$
\begin{aligned}
\frac{\partial p}{\partial t}= & c_{\mathrm{hy}} \frac{\partial^{2} p}{\partial x_{2}^{2}}+\Lambda \frac{\partial T}{\partial t}-\frac{\beta}{\beta^{\star}} \frac{\partial \gamma^{\mathrm{p}}}{\partial t} \\
& +\frac{\rho_{\mathrm{s}}-\rho_{\mathrm{f}} \zeta}{\rho_{\mathrm{f}} \beta^{\star}} \frac{A_{0}}{M_{\mathrm{CaCO}_{3}}} \mathrm{e}^{-T_{\mathrm{c}} / T}
\end{aligned}
$$

where $\rho_{\mathrm{s}}$ is the density of the solid skeleton, $\rho_{\mathrm{f}}$ is the density of the fluid phase, $c_{\text {hy }}$ is the hydraulic diffusivity, $\beta^{*}$ is the storage capacity and $\Lambda=\left(\lambda_{\mathrm{f}}-\lambda_{\mathrm{n}}\right) / \beta^{*}$ is the pressurisation coefficient, which is expressed as the difference of the thermal expansion coefficients of the fluid $\left(\lambda_{\mathrm{f}}\right)$ and the pore volume $\left(\lambda_{\mathrm{n}}\right)$. Notice that $\lambda_{\mathrm{n}}$ is equal to the elastic thermal expansion coefficient of the solid fraction $\alpha_{\mathrm{s}}$ (Sulem, 2010), that the value of $\Lambda$ is assumed in the literature to vary from $0 \cdot 1 \mathrm{MPa} /{ }^{\circ} \mathrm{C}$ (Vardoulakis, 2002) to $1.5 \mathrm{MPa} /{ }^{\circ} \mathrm{C}$ (Rice, 2006) and that the coefficient $\zeta$ represents a concentration ratio of the products of the reaction, calculated for the calcite decomposition to be $1 \cdot 24$ (Veveakis et al., 2012).

By also assuming that all the mechanical work input is converted into heat and that the heat is expressed through Fourier's law, we may obtain the diffusion-reaction 
temperature equation (Sulem \& Famin, 2009; Veveakis et al., 2012)

$$
\frac{\partial T}{\partial t}=c_{\text {th }} \frac{\partial^{2} T}{\partial x_{2}^{2}}+\frac{1}{\rho C} \tau \frac{\partial \gamma^{\mathrm{p}}}{\partial t}-\frac{|\Delta H|}{\rho C} \frac{\rho_{\mathrm{s}}}{M_{\mathrm{CaCO}_{3}}} A_{0} \mathrm{e}^{-T_{\mathrm{c}} / T}
$$

where $c_{\text {th }}$ is the thermal diffusivity, $\rho C$ is the specific heat capacity of the mixture and $|\Delta H| \approx E$ is the specific enthalpy of the reaction, expressing the energy consumed during an endothermic reaction.

\section{Boundary conditions}

At the boundaries of the shear zone of Fig. 1, conditions for displacements, rotations, pore pressure and temperature need to be imposed. To this end, constant values are assumed for the applied stresses and couple stresses, leading through the constitutive laws to Dirichlet (constant) boundary conditions for the displacements and rotations. For the pore pressure and temperature, we identify the timescales of diffusion over the width $D$ as the time at which any pore pressure and temperature variations will be diffused away from the shear band

$$
\begin{aligned}
& t_{\text {hy }}=D^{2} / c_{\text {hy }} \\
& t_{\text {th }}=D^{2} / c_{\text {th }}
\end{aligned}
$$

In addition, the timescale of imposing pore pressure and temperature variations is the timescale of the mechanical input, determined by the boundary shear strain rate $\dot{\gamma}_{\mathrm{b}}$ as (Garagash \& Rudnicki, 2003a, 2003b)

$$
t_{\text {mech }}=1 / \dot{\gamma}_{\mathrm{b}}
$$

When $t_{\text {mech }} \gg t_{\text {hy,th }}$, the mechanical input is perturbing the thermal and hydraulic flow in slower timescales than those of diffusion. This means that the system has time to diffuse away all the imposed variations and maintain constant boundary values for temperature and pore pressure. At the other extreme, when $t_{\text {mech }} \ll t_{\text {hy,th }}$, new mechanical input is being accumulated to the system, not having enough time to diffuse away perturbations. Hence, thermal and fluid fluxes are trapped inside the shear band, in a limit that is called undrained-adiabatic (Vardoulakis, 2002; Rice, 2006).

Typical values of the thermal and hydraulic diffusivity are of the order of $10^{-5}-10^{-6} \mathrm{~m}^{2} / \mathrm{s}$ for impermeable (permeability $k<10^{-18} \mathrm{~m}^{2}$ ) soils saturated in water (Sulem \& Famin, 2009). This value would mean that the undrained-adiabatic limit would be reached once the shear band is sheared with rates $\dot{\gamma}_{\mathrm{b}} \gg 1$ for a shear zone $1 \mathrm{~mm}$ thick. This limit is of particular interest in the mechanics of faults, where cataclastic (finely granulated) shear zones saturated in fluids experience dynamic stress drops during co-seismic slip at such strain rates (Rice, 2006; Sulem \& Famin, 2009).

Based on this, this study is restricted to the undrainedadiabatic limit and the values of the fluid and heat fluxes at the boundary of the layer are fixed equal to zero. Following the previous discussion, fluid and heat flows inside the layer are permitted and the minimum thickness of the layer is back-calculated for this assumption to be valid.

\section{UNDRAINED-ADIABATIC ANALYSIS OF A CAUCHY CONTINUUM}

In the case of a Cauchy elasto-plastic material and in the absence of inertia terms, we may linearise the system of equations and induce a perturbation (denoted as tilded fields (e.g. $\tilde{p})$ ) around its steady state value, to obtain

$$
\begin{aligned}
& \frac{\partial \tilde{p}}{\partial t}=c_{\mathrm{hy}} \frac{\partial^{2} \tilde{p}}{\partial x_{2}^{2}}+\Lambda \frac{\partial \tilde{T}}{\partial t}-\frac{\beta}{\beta^{\star}} \frac{\partial \tilde{\gamma}^{\mathrm{p}}}{\partial t}+\frac{\rho_{\mathrm{s}}-\rho_{\mathrm{f}} \zeta}{\rho_{\mathrm{f}} \beta^{\star}} \frac{A_{0}}{M_{\mathrm{CaCO}_{3}}} \frac{\tilde{T}}{T_{\mathrm{c}}} \\
& \frac{\partial \tilde{T}}{\partial t}=c_{\mathrm{th}} \frac{\partial^{2} \tilde{T}}{\partial x_{2}^{2}}+\frac{1}{\rho C} \tau_{0} \frac{\partial \tilde{\gamma}^{\mathrm{p}}}{\partial t}-\frac{|\Delta H|}{\rho C} \frac{\rho_{\mathrm{s}}}{M_{\mathrm{CaCO}_{3}}} A_{0} \frac{\tilde{T}}{T_{\mathrm{c}}}
\end{aligned}
$$

where $\tau_{0}$ is the steady shear stress (see the detailed method described by Sulem (2010)). Then, the perturbation fields

$$
\frac{\partial \tilde{\gamma}^{\mathrm{p}}}{\partial t}=\frac{\mu}{H} \frac{\partial \tilde{p}}{\partial t}
$$

$$
\tilde{p}=p_{0} \cos \left(\frac{2 \pi x_{2}}{\lambda}\right) \mathrm{e}^{s t}
$$

and

$$
\tilde{T}=T_{0} \cos \left(\frac{2 \pi x_{2}}{\lambda}\right) \mathrm{e}^{s t}
$$

are imposed, $\lambda$ and $s$ being the wavelength and Lyapunov exponent, respectively. The system would then be unstable when $s>0$, or as long as

$$
H<\frac{\Lambda \mu \tau_{0}}{\rho C}-\frac{\mu \beta}{\beta^{\star}}
$$

or

$$
H<\frac{\chi\left(\mu \tau_{0} / \rho C\right)-\left(\mu \beta / \beta^{\star}\right)\left[(2 \pi / \lambda)^{2} c_{\text {th }}+\psi\right]}{(2 \pi / \lambda)^{2}\left(c_{\text {th }}+c_{\text {hy }}\right)+\psi}
$$

in which

$$
\chi=\frac{\rho_{\mathrm{s}}-\rho_{\mathrm{f}} \zeta}{\rho_{\mathrm{f}} \beta^{\star} M_{\mathrm{CaCO}_{3}}} \frac{A_{0}}{T_{\mathrm{c}}}>0
$$

and

$$
\psi=\frac{|\Delta H|}{\rho C} \frac{\rho_{\mathrm{s}}}{M_{\mathrm{CaCO}_{3}}} \frac{A_{0}}{T_{\mathrm{c}}}>0
$$

In the absence of any thermal and chemical effects $(\Lambda=\chi$ $\left.=\psi=c_{\text {th }}=0\right)$, stability is ensured as long as $H>0$. If only thermal diffusion is allowed $\left(\Lambda=\chi=\psi=0\right.$ and $\left.c_{\text {th }} \neq 0\right)$, stability is ensured even for negative values of $H$.

The deficiency of the Cauchy continuum analysis is that instability would set in for zero value of the wavelength, $\lambda$ $=0$, imposing that the undrained-adiabatic shear deformation is localised to a zero thickness band. However, it is well known that the thickness of shear bands is finite and is related to the internal length measures of the material. The Cosserat continuum is a physical starting point for considering microstructure and for developing a theory with internal length measures.

\section{BIFURCATION POINT AND SHEAR BAND THICKNESS AT DIFFERENT TEMPERATURES}

By linearising the resulting system of equations at different temperatures and running standard bifurcation analysis (linear stability analysis), the effect of each of the mechanisms introduced can be identified. At room temperature, where thermal pressurisation and chemical decomposition are inactive, the system is driven by mechanics, providing the well-known results of Muhlhaus \& Vardoulakis (1987). For associative materials under biaxial loading, shear banding will take place at $H=0$, forming a shear band with thickness $D \approx 16 d_{50}, d_{50}$ being 
Table 1. Indicative material parameters for a fault at $7 \mathrm{~km}$ depth, where the initial temperature is about $200^{\circ} \mathrm{C}$

\begin{tabular}{l|l|c|c}
\hline Parameter & Value & Parameter & Value \\
\hline$p$ & $66 \mathrm{MPa}$ & $c_{\text {hy }}$ & $10^{-5} \mathrm{~m}^{2} / \mathrm{s}$ \\
$\sigma_{\mathrm{n}}$ & $200 \mathrm{MPa}$ & $c_{\text {th }}$ & $10^{-6} \mathrm{~m}^{2} / \mathrm{s}$ \\
$M_{\mathrm{CaCO}_{3}}$ & $0 \cdot 1 \mathrm{~kg} / \mathrm{mol}$ & $R \cdot 1 \mathrm{~mm}$ & $10^{4} \mathrm{MPa}$ \\
$\zeta$ & $1 \cdot 24$ & $G$ & $2 \times 10^{4} \mathrm{MPa}$ \\
$\beta$ & 0 & $K$ & $0 \cdot 5$ \\
$\rho_{\mathrm{s}}$ & $2 \cdot 5 \times 10^{3} \mathrm{~kg} / \mathrm{m}^{3}$ & $T_{\mathrm{c}}$ & $800^{\circ} \mathrm{C}$ \\
$\rho_{\mathrm{f}}$ & $10^{3} \mathrm{~kg} / \mathrm{m}^{3}$ & $A_{0}$ & $10^{6} / \mathrm{s}$ \\
$\rho C$ & $2 \cdot 8 \mathrm{MPa} /{ }^{\circ} \mathrm{C}$ & $|\Delta H|$ & $200 \mathrm{~kJ} / \mathrm{mol}$ \\
$\Lambda$ & $0 \cdot 5 \mathrm{MPa} /{ }^{\circ} \mathrm{C}$ & & \\
\hline
\end{tabular}

the mean diameter of the grain size distribution. If pressure sensitive, dilatant medium is considered, the shear band will be formed in the hardening regime $(H>0)$ as shown by Rudnicki \& Rice (1975).

\section{Bifurcation point}

If the temperature is increased in undrained conditions, so that thermal pressurisation is active, Sulem et al. (2011) showed that, even in the absence of dilatancy, the shear band will be formed in the hardening regime $H>0$. If further increase of temperature is induced so that the chemical reaction is triggered, Veveakis et al. (2012) showed that bifurcation is taking place at even higher positive values of $H$.

With these results in mind, linear stability analysis of the Cosserat layer may be carried out. The details of the method are comprehensively presented by Sulem et al. (2011) and Veveakis et al. (2012), allowing application to a $7 \mathrm{~km}$ deep fault with the parameters listed in Table 1. The system is thus linearised at three different temperature regimes

- of the order of the boundary temperature, $T \sim O(1)$

- at higher temperatures where only thermal pressurisation is active, $T \sim O\left(T_{\mathrm{p}}\right)\left(T_{\mathrm{p}}=\sigma_{\mathrm{n}} / \Lambda\right)$

- at elevated temperatures where chemical reaction dominates, $T \sim O\left(T_{\mathrm{c}}\right)$.

The corresponding characteristic equations are solved numerically for each temperature regime, allowing calculation of the critical values of the normalised hardening modulus $h_{\mathrm{cr}}=H_{\mathrm{cr}} /(\sigma+p)$ below which instability sets in. The results are summarised in Table 2 , where different values of the dilatancy $\beta$ are used, from zero to the maximum value $\beta=\mu$ (associative plasticity). We observe that as dilatancy increases the system tends to stabilise since $h_{\mathrm{cr}}$ decreases, as also expected from equation (15). Notice that as the temperature increases, rendering thermal and chemical effects active, the system tends to be prone to instabilities even at the hardening branch of the $\tau-\gamma$ curve, as indicated by the large positive values of $h_{\mathrm{cr}}$.

The dependence of $h_{\mathrm{cr}}$ on the thermal parameters of the problem can be demonstrated at each temperature regime. As shown in Fig. 2(a), at pressurisation temperatures $(T \sim$ $\left.O\left(T_{\mathrm{p}}\right)\right) h_{\mathrm{cr}}$ is sensitive to variations of the thermal pressurisation coefficient $\Lambda$, increasing linearly with $\Lambda$. At higher temperatures, where chemical reaction dominates ( $T$ $\left.\sim O\left(T_{\mathrm{c}}\right)\right) h_{\mathrm{cr}}$ increases with the frequency factor $A_{0}$ of the reaction, obtaining an upper value for strong reactions (Fig. 2(b)).

\section{Shear band thickness}

Stability analysis of the Cosserat layer allows for determination of the shear band thickness. Defined as the critical wavelength number at which instability propagates with maximum finite speed, the analyses performed by Sulem et al. (2011) and Veveakis et al. (2012) revealed that the shear band thickness decreases with increasing temperature, having a lower threshold at $D=3 d_{50}$ at high temperature areas where the chemical reaction is active.

By performing parametric analysis for the shear band thickness at different temperatures, it may be noted that, at $T \sim O\left(T_{\mathrm{p}}\right)$, the shear band thickness decreases hyperbolically with the square root of $\Lambda$ (Fig. 3(a)), being around $150 d_{50}$ at $\Lambda=1$. At the chemical pressurisation regime ( $T$ $\sim O\left(T_{\mathrm{c}}\right)$ ), Veveakis et al. (2012) calculated a bounded hyperbolic reduction with the reaction parameters, up to the lower value $D=3 d_{50}$ (Fig. 3(b)). Since usually $T_{\mathrm{p}} \ll T_{\mathrm{c}}$, as temperature increases due to shear heating, the shear band would shrink in a thermally controlled process (see also Veveakis et al. $(2007,2010))$.

\section{VISCOUS REGULARISATION INDUCED BY MICRO- INERTIA}

The selected wavelength corresponding to the maximum finite propagation velocity of the instability could only be calculated when including the micro-inertia terms on the right-hand sides of equation (8), namely $\rho \frac{\partial v_{1}}{\partial t}, \rho \frac{\partial v_{2}}{\partial t}$ and $\rho \frac{R^{2}}{2} \frac{\partial \dot{\omega}_{\mathrm{c}}}{\partial t}$. Although Cosserat continuum introduces an internal length, thus regularising the spatial ill-posedness of the problem, it cannot regularise temporal instabilities. As depicted in Fig. 4, when the micro-inertia terms are

Table 2. The first three rows correspond to temperatures that are order one with respect to the boundary value. No thermal effects are considered in this case. The next three rows correspond to temperatures near the thermal pressurisation temperatures where the chemical reaction is inactive. The last rows correspond to temperatures near the chemical reaction's activation temperature $T_{\mathrm{c}}$. As dilatancy $\beta$ increases from zero to its maximum value (the friction coefficient $\mu$ ), the system is stabilised as $h_{\mathrm{cr}}$ increases

\begin{tabular}{l|c|c}
\hline Temperature & $\beta$ & $h_{\mathrm{cr}}$ \\
\hline$T \sim O(1)$ & 0 & 0 \\
& $\mu / 10$ & -2 \\
$T \sim O\left(T_{\mathrm{p}}\right)$ & $\mu$ & -15 \\
& 0 & $0 \cdot 015^{\mathrm{a}}$ \\
$T \sim O\left(T_{\mathrm{c}}\right)$ & $\mu / 10$ & $10^{-4}$ \\
& 0 & 0 \\
& $\mu / 10$ & $6^{\mathrm{b}}$ \\
& $\mu$ & $2 \cdot 24$ \\
\hline
\end{tabular}

${ }^{\text {a }}$ Sulem et al. (2011)

${ }^{\mathrm{b}}$ Veveakis et al. (2012) 

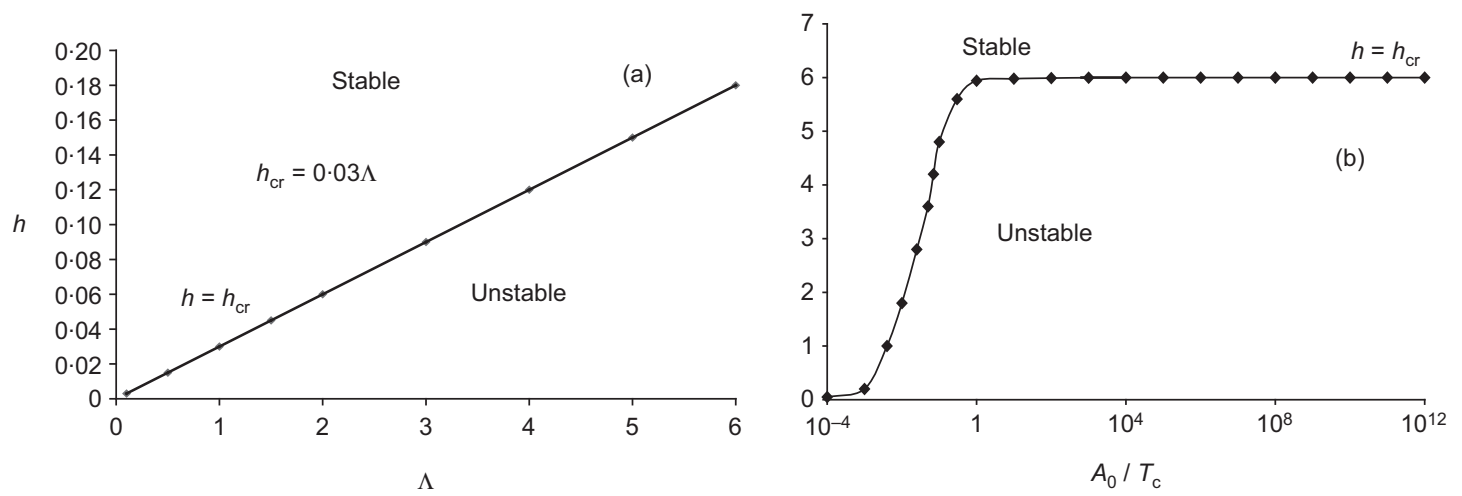

Fig. 2. Evolution of $h_{\mathrm{cr}}$ : (a) at $T \sim O\left(T_{\mathrm{p}}\right)$ as a function of the pressurisation coefficient; (b) at $T \sim O\left(T_{\mathrm{c}}\right)$ as a function of the reaction parameters $A_{0}$ and $T_{\mathrm{c}}$ (Reprinted from Journal of Structural Geology, 38, E. Veveakis, J. Sulem and I. Stefanou, Modeling of fault gouges with Cosserat continuum mechanics: Influence of thermal pressurization and chemical decomposition as coseismic weakening mechanisms. pp. 254-264, Copyright (2012), with permission from Elsevier.)
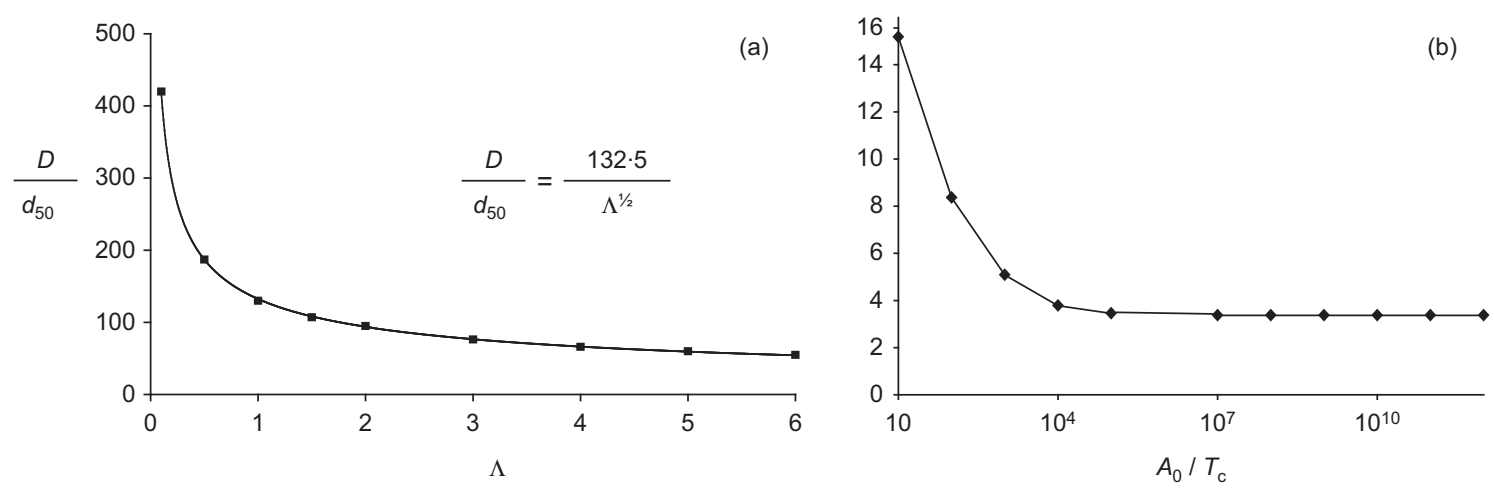

Fig. 3. Evolution of the shear band thickness: (a) at $T \sim O\left(T_{\mathrm{p}}\right)$ as a function of the pressurisation coefficient; (b) at $T \sim O\left(T_{\mathrm{c}}\right)$ as a function of the reaction parameters $A_{0}$ and $T_{\mathrm{c}}$ (Reprinted from Journal of Structural Geology, 38, E. Veveakis, J. Sulem and I. Stefanou, Modeling of fault gouges with Cosserat continuum mechanics: Influence of thermal pressurization and chemical decomposition as coseismic weakening mechanisms. pp. 254-264, Copyright (2012), with permission from Elsevier.)

neglected, thus assuming stress equilibrium at the Cosserat scale, the shear band forms with infinite propagation velocity in clear evidence of ill-posedness of the mathematical system. This ill-posedness is alleviated once the microinertia terms are introduced, providing finite propagation velocities of the instability.

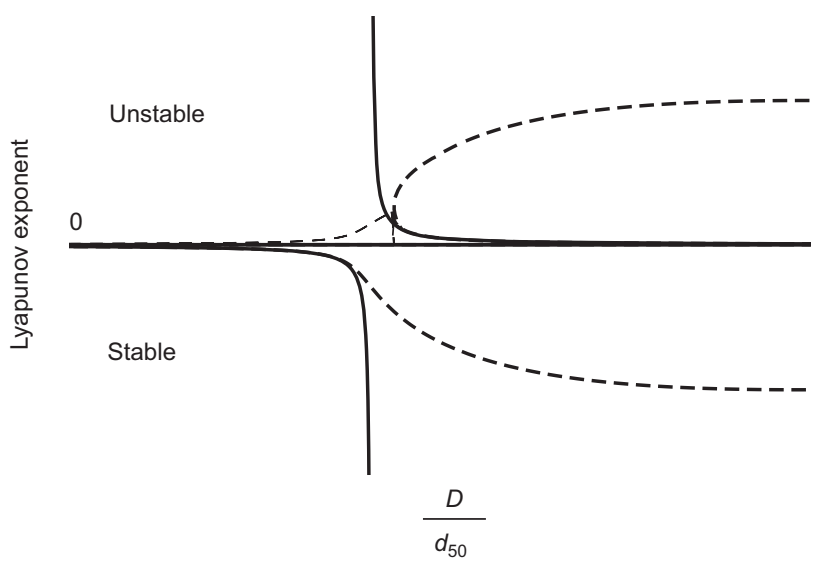

Fig. 4. Real part of the roots of the characteristic equation when the Cosserat inertia terms are neglected (solid curve) and included (dashed curve). Notice that in the absence of inertia terms, the growth coefficient (Lyapunov exponent) is infinite for finite wavelength (Sulem et al., 2011), implying that instability propagates at infinite velocities
This regularisation technique provides identical results to the viscous regularisation frequently used by the community to regularise in time the ill-posed momentum balance equation of a saturated Cauchy continuum (Vardoulakis, 2002). With viscous regularisation, a characteristic time (rate) from the microstructure is introduced in the constitutive law of plasticity. For regularising a Mohr-Coulomb failure criterion for a Cauchy continuum, Vardoulakis (2002) showed that the friction coefficient $\mu$ should be allowed to vary with the plastic strain rate and the second gradient of it

$$
\mu=\mu\left(\dot{\gamma}^{\mathrm{p}}\right)-\eta l_{\mathrm{c}}^{2} \nabla^{2} \dot{\gamma}^{\mathrm{p}}
$$

where $\eta\left(=\partial \mu / \partial \dot{\gamma}^{\mathrm{p}}\right)$ is a rate sensitivity parameter and $l_{\mathrm{c}}$ a characteristic material length imposed by the regularisation. Noting that this technique not only imposes viscosity in the material, but also actually influences its constitutive response, inducing rate dependency, it can be inferred that the translational and rotational inertia terms at the grain scale (Cosserat micro-inertia) could be the origin of possible rate dependency effects in saturated granular media under simple shear.

\section{CONCLUSIONS}

This study generalised the classical mechanical concept of failure for geomaterials to account for changes imposed by thermo-hydro-chemical feedbacks. The following conclusions are drawn. 
- Failure during shear occurs at higher positive values of the hardening modulus as temperature increases, due to thermal pressurisation or chemical pressurisation.

- Apart from mechanics, shear band thickness depends on temperature, localising with increasing temperature. Hence, one may envision that in a real process where shear deformation would produce heat due to friction, considering all temperatures up to the reaction's activation energy, the problem could potentially have several distinct length scales spanning from a purely mechanical to a chemo-mechanical band at elevated temperatures.

- Micro-inertia due to grain translation and rotation introduces the necessary rate effects to regularise the system during simple shear. Since rate dependency during shearing of cohesionless granular media with non-crushable grains was shown to be necessary to regularise the problem of a Cauchy continuum, we suggest that this rate dependency may originate from the micro-inertia of the grains. This suggestion remains to be proven rigorously, a proof that is beyond the scope of the present analysis.

\section{REFERENCES}

Garagash, D. I. \& Rudnicki, J. W. (2003a). Shear heating of a fluid-saturated slip-weakening dilatant fault zone, 1, limiting regimes. J. Geophys. Res. 108, No. B2, 2121.

Garagash, D. I. \& Rudnicki, J. W. (2003b). Shear heating of a fluid-saturated slip-weakening dilatant fault zone, 2, quasidrained regime. J. Geophys. Res. 108, No. B10, 2472.

Hill, R. (1962). Acceleration waves in solids. J. Mech. Phys. Solids 10, No. 1, 1-16.
Mandel, J. (1966). Conditions de stabilite et postulate de Drucker. In Rheology and soil mechanics (IUTAM symposium) (Kravtchenko, J. \& Sirieys, P. M. (eds)). Berlin/Heidelberg: Springer Verlag, pp. 58-67.

Muhlhaus, H. \& Vardoulakis, I. (1987). Thickness of shear bands in granular materials. Géotechnique 37, No. 3, 271-283.

Rice, J. R. (2006). Heating and weakening of faults during earthquake slip. J. Geophys. Res. 111, No. B5, 311.

Rudnicki, J. \& Rice, J. (1975). Conditions for the localization of deformation in pressure sensitive dilatant materials. J. Mech. Phys. Solids 23, 371-394.

Sulem, J. (2010). Bifurcation theory and localization phenomena. Eur. J. Environ. Civil Engng 14, No. 8-9, 989-1009.

Sulem, J. \& Famin, V. (2009). Thermal decomposition of carbonates in fault zones: slip-weakening and temperaturelimiting effects. J. Geophys. Res. 114, No. B3, 309.

Sulem, J., Stefanou, I. \& Veveakis, E. (2011). Stability analysis of undrained adiabatic shearing of a rock layer with Cosserat microstructure. Gran. Matter 13, No. 3, 261-268.

Vardoulakis, I. (2002). Dynamic thermo-poro-mechanical analysis of catastrophic landslides. Géotechnique 52, No. 3, 157171.

Vardoulakis, I. \& Sulem, J. (eds) (1995). Bifurcation analysis in geomechanics. London: Blackie Academic \& Professional.

Veveakis, E., Vardoulakis, I. \& Toro, G. D. (2007). Thermoporomechanics of creeping landslides: The 1963 Vaiont slide, northern Italy. J. Geophys. Res. 112, No. F3, 26.

Veveakis, E., Alevizos, S. \& Vardoulakis, I. (2010). Chemical reaction capping of thermal instabilities during shear of frictional faults. J. Mech. Phys. Solids 58, 1175-1194.

Veveakis, E., Sulem, J. \& Stefanou, I. (2012). Modeling of fault gouges with cosserat continuum mechanics: Influence of thermal pressurization and chemical decomposition as coseismic weakening mechanisms. J. Struct. Geol. 38, 254-264.

\section{WHAT DO YOU THINK?}

To discuss this paper, please email up to 500 words to the editor at journals@ice.org.uk. Your contribution will be forwarded to the author(s) for a reply and, if considered appropriate by the editorial panel, will be published as a discussion. 